Kompass

Onkologie

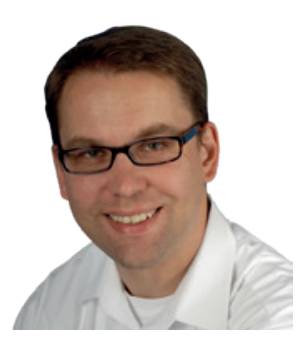

\section{Christoph Hoeller}

Universitätsklinik für Dermatologie, Medizinische Universität Wien, Wien, Österreich

\title{
Melanom: Offene Fragen aktueller Therapiestandards werden sukzessive aufgearbeitet
}

Sehr geehrte Kolleginnen und Kollegen! Die Behandlung des Melanoms hat sich in den letzten 10 Jahren von Grund auf verändert. Die Therapie mit PD-1 blockierenden Antikörpern, die Kombination aus dem PD-1 Inhibitor Nivolumab und dem CTLA-4 Inhibitor Ipilimumab, sowie die Kombination von aus BRAF und MEK Inhibitoren sind die aktuellen klinischen Standards für die Immun- bzw. zielgerichtete Therapie beim inoperabel metastasierten Melanom. Insbesondere in der Entwicklung der ImmunCheckpoint Inhibitoren diente das Melanom dabei als Modelltumor. Rezente Langzeitdaten zeigen eindrucksvoll, wie sehr sich durch diese Therapien die Situation unserer Patienten geändert hat. Waren in den Zeiten der Chemotherapie nach 5 Jahren nur noch zwischen 3-5\% aller Patienten mit inoperabler Fernmetastasierung am Leben, so sind dies heute unter der Kombination von Ipilimumab und Nivolumab über 50\% der Patienten. Ebenso haben die Langzeitdaten dieser Studien aber auch gezeigt, dass im allgemeinen Patienten, welche als erste Therapielinie eine PD-1 Antikörper basierte Therapie erhalten, langfristig ein besseres Outcome haben, als Patienten welche eine BRAF/MEK Inhibitor Therapie in der Erstlinie erhalten. Sowohl die Guidelines der EADO/EORTC [1] als auch die aktuellen ESMO Guidelines [2] empfeh- len daher als Erstlinientherapie beim metastasierten Melanom eine Immuntherapie mit Checkpoint-Inhibitoren.

Auch im adjuvanten Setting nach kompletter Operation einer lokoregionären Metastasierung zeigen sowohl die PD-1 Antikörper Nivolumab und Pembrolizumab als auch die BRAF/MEK Inhibitor Kombination Dabrafenib/Trametinib, letztere natürlich nur bei BRAF mutierten Patienten, in etwa eine Halbierung der Rückfallrate, wodurch vielen Patienten der Übergang in ein fernmetastasiertes Stadium erspart werden kann. Dr. Jan Maschke wird in diesem Heft über einen interessanten Abstract zum Zusammenhang von immunvermittelten Nebenwirkungen und dem rückfallfreien Überleben aus einer adjuvanten Studie mit Pembrolizumab [3] berichten. Dass eine adjuvante Therapie auch nach kompletter Resektion von Fernmetastasen hoch effektiv sein kann, wurde rezent in der dreiarmigen ImmunNED Studie der DeCOG gezeigt [4]. Hier erhielten Patienten mit einem komplett resezierten Stadium IV Melanom randomisiert entweder Nivolumab oder Nivolumab und Ipilimumab. Der Kontrollarm erhielt ein Placebo mit der Option eines Cross-overs bei Progress. Während nur 14\% der Patienten im Kontrollarm nach 24 Monaten keinen Relapse aufwiesen, waren dies $42 \%$ im Nivolumab- und beeindruckende $70 \%$ im Kombinationsarm.
Aktuell wird neben adjuvanten Studien, in welche bereits Patienten mit einem primären Melanom mit hohem Rezidivrisiko, also Patienten ohne Metastasierung, eingeschlossen werden auch sehr stark an der Etablierung eines neoadjuvanten Therapieansatzes für Patienten mit klinisch detektierten - «makroskopischen» - lokoregionären Lymphknotenmetastasen gearbeitet. In Kooperation mehrerer Zentren, dem «International Neoadjuvant Melanoma Consortium» (https://melanoma-inc.org/) konnte gezeigt werden, dass auch in dieser Situation die Kombination aus gegen PD-1 und CTLA-4 gerichteten Antikörpern die höchste Effektivität aufweist, wobei sich vor allem die Rate an pathologischer kompletter oder nahezu kompletter Remission im OP-Präparat als maßgeblicher prädiktiver Parameter etabliert hat. Initial war eine solche Therapie aber von extrem hoher Toxizität mit bis zu 90\% Grad 3 und 4 Nebenwirkungen nach den CTC Kriterien begleitet. Dies konnte jedoch durch eine Dosismodifikation in der OpACIN-neo Studie [5], über welche Dr. Alexander Thiem in diesem Heft berichten wird, rezent gelöst werden. Der Weg für eine randomisierte Studie im Vergleich zum aktuellen Standard der postoperativen adjuvanten Therapie wurde so geebnet. Während also für die Mehrheit der Melanom-Patientinnen und Patienten heute 
deutlich effektivere Therapien zur Verfügung stehen, gibt es allerdings nach wie vor seltenere Subgruppen von Melanomen, die entweder in deutlich geringerem Ausmaß auf diese Therapien ansprechen, bzw. für die solche Therapien mangels entsprechender genetischer Veränderungen gar nicht in Frage kommen, oder aufgrund fehlender Erfahrung bei Mutationen außerhalb der typischen «hot-spots» nicht verwendet werden. Hierzu finden Sie in dieser Ausgabe einerseits einen interessanten Case Report einer seltenen Variante einer BRAF Mutation [6].

Wesentlich von dieser Problematik betroffen sind aber Patienten mit extra-kutanen Melanomen. Diese unterscheiden sich durch die Art ihrer Entstehung. Es ist nur sehr selten eine UV-Genese vorhanden,

\section{Literatur}

1 Garbe C, Amaral T, Peris K, Hauschild A, Arenberger P, Bastholt L, et al. European consensus-based interdisciplinary guideline for melanoma. Part 2:Treatment - Update 2019. Eur J Cancer. 2020;126:159-77.

2 Michielin O, van Akkooi ACJ, Ascierto PA, Dummer R, Keilholz U, clinicalguidelines@ esmo.org EGCEa. Cutaneous melanoma: ESMO Clinical Practice Guidelines for diagnosis, treatment and follow-updagger. Ann Oncol. 2019;30(12):1884-901.

3 Eggermont AMM, Kicinski M, Blank CU, Mandala M, Long GV, Atkinson V, et al. Association Between Immune-Related Adverse Events and Recurrence-Free Survival Among Patients With Stage III Melanoma Random- was zu anderen genetischen Veränderungen führt, für die keine oder nur weniger aktive, gezielte Therapien zur Verfügung stehen. Diese Tumore weisen typischerweise auch eine deutlich reduzierten Mutationslast im Tumorgenom, einem der möglichen prädiktiven Parameter für das Ansprechen auf eine Immuntherapie, auf. Besonders schwierig ist die Situation beim uvealen Melanom, worauf Dr. Anja Wessely in einem Bericht zu einer Übersichtsarbeit zu diesem Thema eingeht [7].

Ein möglicher Weg auch für diese Subgruppen des Melanoms effektivere Therapie entwickeln zu können, ist diese Tumoren möglichst detailliert genetisch aufzuarbeiten. Ein sehr gutes Beispiel dafür ist die letzten Sommer in Nature erschienene Publikation einer Arbeitsgruppe um Rick Scolyer vom Melanoma Insitute of Australia, in der die genetischen Veränderungen von Schleimhautmelanomen detailliert aufgearbeitet wurden (8). Hierbei zeigten sich neben bekannten Mutationen Hinweise darauf, dass viele dieser Tumoren auch einer Therapie mit CDK4/6-Inhibitoren zugänglich sein könnten, welche bislang noch keine Zulassung bei Melanomen hat.

Die vielen neuen Standards, die in der Therapie des Melanoms in den letzten 10 Jahren etabliert wurden, haben eine Vielzahl neuer Fragen aufgeworfen. Diese Fragen werden nun sukzessive auf höchstem wissenschaftlichem Niveau aufgearbeitet. Die neu gewonnenen Daten bilden die Grundlage für die weitere Verbesserung und Erweiterung der Therapie des Melanoms. ized to Receive Pembrolizumab or Placebo: A Secondary Analysis of a Randomized Clinical Trial. JAMA Oncol. 2020.

4 Zimmer L, Livingstone E, Hassel JC, Fluck M, Eigentler $\mathrm{T}$, Loquai $\mathrm{C}$, et al. Adjuvant nivolumab plus ipilimumab or nivolumab monotherapy versus placebo in patients with resected stage IV melanoma with no evidence of disease (IMMUNED): a randomised, double-blind, placebo-controlled, phase 2 trial. Lancet. 2020;395(10236):1558-68.

5 Rozeman EA, Menzies AM, van Akkooi ACJ, Adhikari C, Bierman C, van de Wiel BA, et al. Identification of the optimal combination dosing schedule of neoadjuvant ipilimumab plus nivolumab in macroscopic stage III mel- anoma (OpACIN-neo): a multicentre, phase 2 , randomised, controlled trial. Lancet Oncol 2019;20(7):948-60.

6 Bjursten S, Vannas C, Filges S, Puls F, Pandita A, Fagman H, et al. Response to BRAF/MEK Inhibition in A598_T599insV BRAF Mutated Melanoma. Case Rep Oncol. 2019;12(3):872-9.

7 Wessely A, Steeb T, Erdmann M, Heinzerling L, Vera J, Schlaak M, et al. The Role of Immune Checkpoint Blockade in Uveal Melanoma. Int J Mol Sci. 2020;21(3).

8 Newell F, Kong Y, Wilmott JS, Johansson PA, Ferguson PM, Cui $\mathrm{C}$, et al. Whole-genome landscape of mucosal melanoma reveals diverse drivers and therapeutic targets. Nat Commun. 2019;10(1):3163. 